\title{
Intravenous patient-controlled analgesia hydromorphone combined with pregabalin for the treatment of postherpetic neuralgia: a multicenter, randomized controlled study
}

\author{
Ying Huang ${ }^{1, *}$, Chenjie Xü ${ }^{2, *}$, Tao Zeng ${ }^{3, *}$, Zhongming $\mathrm{Li}^{4}$, Yanzhi Xia ${ }^{5}$, Gaojian Tao ${ }^{1}$, Tong Zhu ${ }^{1}$, Lijuan Lu$^{1}$, \\ Jing $\mathrm{Li}^{1}$, Taiyuan Huang ${ }^{1}$, Hongbo Huai ${ }^{1}$, Benxiang $\mathrm{Ning}^{1}$, Chao $\mathrm{Ma}^{1}$, Xinxing Wang ${ }^{6}$, Yuhua Chang ${ }^{7}$, \\ Peng $\mathrm{Mao}^{6}$, and Jian Lin ${ }^{1}$
}

'Department of Pain, Drum Tower Hospital, Medical School of Nanjing University, Nanjing, Jiangsu, China

${ }^{2}$ Department of Anesthesiology and Pain, Nanjing First Hospital, Nanjing Medical University, Nanjing, Jiangsu, China

${ }^{3}$ Department of Pain, Kunshan Hospital of Integrated Traditional Chinese and Western Medicine, Kunshan, Jiangsu, China

${ }^{4}$ Department of Pain, Affiliated Hospital of Nantong University, Nantong, Jiangsu, China

${ }^{5}$ Department of Anesthesiology, Shanghai Public Health Clinical Center, Fudan University, Shanghai, China

${ }^{6}$ Department of Pain Medicine, China-Japan Friendship Hospital, Beijing, China

${ }^{7}$ Department of Pain, Bayingolin Mongolian Autonomous Prefecture People's Hospital, Xinjiang, China

Received October 20, 2020

Revised December 13, 2020

Accepted December 28, 2020

Handling Editor: Young-Bok Lee

Correspondence

Jian Lin

Department of Pain, Drum Tower Hospital, Medical School of Nanjing University, 321 Zhongshan Road, Nanjing, Jiangsu 210008, China

Tel: +8625-83106666, Fax: +8625-83106666

E-mail: linjian419@njglyy.com

\section{Peng Mao}

Department of Pain Medicine, ChinaJapan Friendship Hospital, 2 Yinghuayuan East Street, Beijing 100029, China Tel: +8610-84205288, Fax: +8610-84205288

E-mail: doctormaopeng@126.com

Yuhua Chang

Department of Pain, Bayingolin Mongolian Autonomous Prefecture People's Hospital, 56 Renmin East Road, Korla, Xinjiang 841000 , China

Tel: +86996-2021856, Fax: +86996-2021856

E-mail: 1491955033@qq.com

*These authors contributed equally to this work.
Background: Postherpetic neuralgia (PHN) is the most common complication of acute herpes zoster. The treatment of PHN remains a challenge for clinical pain management. Despite the effectiveness of anticonvulsants, antidepressants, and lidocaine patches in reducing PHN, many patients still face intractable pain disorders. In this randomized controlled study, we evaluated whether hydromorphone through intravenous patient-controlled analgesia (IV PCA) was effective in relieving PHN. Methods: Patients with PHN were randomly divided into two groups, one group received oral pregabalin with IV normal saline, another group received oral pregabalin with additional IV PCA hydromorphone for two weeks. Efficacy was evaluated at 1, 4, and 12 weeks after the end of the treatments.

Results: Two hundred and one patients were followed up for 12 weeks. After treatment, numerical rating scale (NRS) score of patients in the hydromorphone group was significantly lower than that of the control group, and the difference of NRS scores between the two groups was statistically significant at 4 and 12 weeks after treatment. The frequency of breakthrough pain in the hydromorphone group was significantly lower than that in the control group 1 and 4 weeks after treatment. After treatment, the quality of sleep in the hydromorphone group was significantly improved compared with the control group. The most common adverse reactions in the hydromorphone group were dizziness and nausea, with no significant respiratory depression.

Conclusions: IV PCA hydromorphone combined with oral pregabalin provides superior pain relief in patients with $\mathrm{PHN}$, which is worthy of clinical application and promotion.

Key Words: Analgesia, Patient-Controlled; Analgesics, Opioid; Anticonvulsants; Hydromorphone; Neuralgia, Postherpetic; Opiate Alkaloids; Pain, Intractable; Pain Management; Pregabalin. (a) This is an open-access article distributed under the terms of the Creative Commons Attribution Non-Commercial License (http://creativecommons.org/licenses/by-nc/4.0/), which permits unrestricted non-commercial use, distribution, and reproduction in any medium, provided the original work is properly cited.

(C) The Korean Pain Society, 2021
Author contributions: Ying Huang: Writing/manuscript preparation; Chenjie Xu: Writing/manuscript preparation; Tao Zeng: Writing/manuscript preparation; Zhongming Li: Investigation; Yanzhi Xia: Investigation; Gaojian Tao: Investigation; Tong Zhu: Data curation; Lijuan Lu: Data curation; Jing Li: Supervision; Taiyuan Huang: Supervision; Hongbo Huai: Investigation; Benxiang Ning: Investigation; Chao Ma: Investigation; Xinxing Wang: Investigation; Yuhua Chang: Supervision; Peng Mao: Project administration; Jian Lin: Supervision. 


\section{INTRODUCTION}

Postherpetic neuralgia (PHN) is zoster-related neuralgia that is still present one month after the development of skin lesions [1]. The prevalence of PHN increases with age, between 25 to 50 percent of patients with herpes zoster (HZ) older than 50 years of age develop PHN, and up to 75 percent of patients with $\mathrm{HZ}$ older than 70 years of age develop PHN [2-4]. The pain can last for weeks or longer, and can last for many years, severely affecting patients' sleep and other daily activities [5].

Antiviral therapy can reduce the severity and duration of HZ, but cannot prevent PHN [6]. Pharmacological therapy is the basis of PHN treatment [7]. The U.S. Food and Drug Administration has approved some drugs for PHN, including pregabalin, gabapentin, capsaicin patches, and lidocaine patches [1]. But there are still some patients whose pain is not well controlled. Although various procedures aimed at pain relief for PHN have been devised and tested, no established treatment for PHN has yet been identified [8].

Hydromorphone is a semi-synthetic derivative of morphine, which plays an analgesic role in the central nervous system through the excitation of $\mu$-opioid receptors [9]. Hydromorphone is about five to seven times more effective at relieving pain than morphine [10]. Although hydromorphone has been used since 1975 to treat severe pain [11], and opioids have been reported in PHN [12], the use of hydromorphone in PHN has not been reported. Opioids are often administered according to a schedule rather than on-demand, which can lead to inadequate analgesia for patients with PHN. In this study, intravenous patientcontrolled analgesia (IV PCA) was used to treat PHN. The purpose of this randomized controlled clinical trial was to evaluate the efficacy of pregabalin combined with IV PCA hydromorphone in patients with PHN and its impact on their quality of life, as well as to evaluate adverse drug reactions and complications.

\section{MATERIALS AND METHODS}

This study is a prospective, multicenter, randomized controlled clinical trial. The protocol of this study was approved by the Human Ethics Committee of Nanjing Drum Tower Hospital and implemented in accordance with the Declaration of Helsinki (approval number: 2018-136-02). Informed consent was obtained from all patients prior to the study. The clinical trial was registered with www.chictr.org.cn on December 5, 2018 (No. ChiCTR1800019880). All participants were treated from December 6, 2018 to October 30, 2019 and provided informed written consent.

\section{Patients}

As no data were available regarding the effect of hydromorphone on PHN patients, sample size was estimated that, to achieve $90 \%$ power at the 0.05 significance level with a standard deviation of $20 \%$, a total of 200 patients were required to detect a difference of $15 \%$ in change from baseline in numerical rating scale (NRS) scores between the hydromorphone and control groups. From December 6, 2018, to October 30, 2019, 201 patients in 7 hospitals, including Nanjing Drum Tower Hospital, Nanjing First Hospital, Kunshan Hospital of Integrated Traditional Chinese and Western Medicine, Affiliated Hospital of Nantong University, Shanghai Public Health Clinical Center, China-Japan Friendship Hospital, and Bayingolin Mongolian Autonomous Prefecture People's Hospital were included in this clinical trial (Fig. 1). We defined PHN as HZ-associated neuralgia lasting for more than one month. The inclusion criteria were: being at least 18 years old with a diagnosis of PHN, an average NRS score above 4, and willingness to comply with assigned treatment and followup measurements. Exclusion criteria were: significant pain from causes other than PHN, abnormal coagulation, mental illness, substance abuse, previous hypersensitivity to hydromorphone, pregnancy during the study or plans to become pregnant, lack of cooperation, and severe systemic, metabolic, or neurological disease.

\section{Procedures}

Two hundred and one patients were randomly assigned to one of the following treatment groups: The control group received PHN conventional treatment oral pregabalin (75 mg Lyrica; Pfizer, New York, NY) 75-150 mg bid with IV

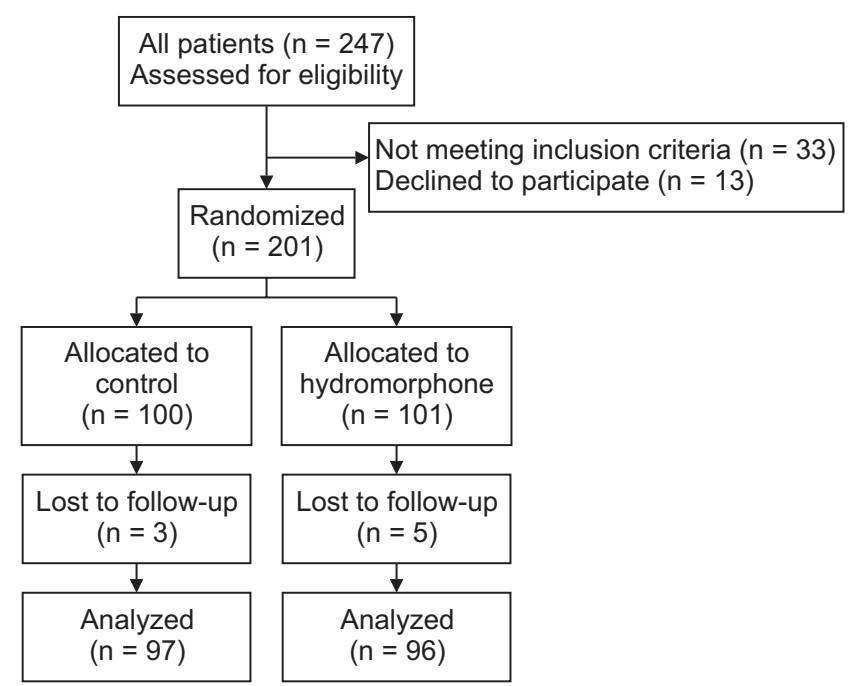

Fig. 1. Assignment of patients to treatment groups. 
normal saline (NS) $(2 \mathrm{~mL} / \mathrm{h})$. During the study period, the use of narcotic analgesics was prohibited. The hydromorphone group, in addition to oral pregabalin, was treated with an IV PCA hydromorphone (2 mg:2 mL, Ruining; Yichang Humanwell Pharmaceutical, Yichang, China)based dose of $2 \mathrm{mg} / \mathrm{d}$. Patients can add a $0.1 \mathrm{mg}$ bolus with a lockout time of 15 minutes according to their level of pain. The bolus dose was recorded every 24 hours and then increased to the base dose. The treatment course lasted two weeks.

\section{Efficacy measures}

A computer program was used to randomize patients. Patients were evaluated before randomization, as well as 1 week, 4 weeks, and 12 weeks after treatment. We collected all the demographic and clinical data, including age, sex, duration of pain, location of pain, severity of pain, breakthrough pain, and quality of life. Breakthrough pain is a transient, short-term burst of pain. Patients were asked to quantify their average pain with a NRS. A score of 0 means no pain, and a score of 10 means the most severe pain imaginable [13]. Quality of life was assessed using the Short-Form McGill Pain Questionnaire (SFMPQ) and Pittsburgh sleep quality index (PSQI). The SFMPQ contains 15 descriptors (4 affective descriptors and 11 sensory descriptors), including 4 intensity scales ( 0 for none and 3 for severe) [14]. A total score was derived from the sum of responses to all 15 descriptors. The PSQI scores range from 0 to 21, with higher scores indicating worse sleep quality [15]. If the patient is not able to return to the pain clinic for a follow-up visit, the follow-up was conducted by telephone. All adverse events and complications associated with hydromorphone, including nausea, vomiting, pruritus, dizziness, drowsiness, constipation, difficulty passing urine, sweating, and respiratory depression, were documented throughout the study.

\section{Statistical analysis}

All statistical analyses were performed using SPSS statistics ver. 20 (IBM Corp., Armonk, NY). Two-tailed Z tests were used to verify that the 2 groups were balanced in terms of demographic and clinical variables (sex, age, severity of pain, localization of pain, and duration of pain). Changes in pain intensity over time were analyzed with a repeated measures analysis of variance (ANOVA). NRS pain scores were also compared between the 2 groups using repeated-measures ANOVA. Significance was assessed at $P<0.05$.
Table 1. Demographic characteristics of the study participants

\begin{tabular}{lccc}
\hline \multicolumn{1}{c}{ Characteristic } & $\begin{array}{c}\text { Control group } \\
(\mathrm{n}=97)\end{array}$ & $\begin{array}{c}\text { Hydromorphone } \\
\text { group }(\mathrm{n}=96)\end{array}$ & $P$ value \\
\hline $\begin{array}{l}\text { Age }(\mathrm{yr}) \\
\text { Sex }\end{array} \quad 66.6 \pm 14.9$ & $67.2 \pm 9.6$ & 0.501 \\
$\quad$ Male & $51(52.6)$ & $46(47.9)$ & 0.421 \\
$\quad$ Female & $46(47.4)$ & $50(52.1)$ & 0.413 \\
Localization & & & \\
$\quad$ Head & $11(11.3)$ & $13(13.6)$ & 0.235 \\
$\quad$ Cervial & $15(15.5)$ & $17(17.7)$ & 0.322 \\
$\quad$ Thoracic & $45(46.4)$ & $39(40.6)$ & 0.438 \\
$\quad$ Lumbar & $26(26.8)$ & $27(28.1)$ & 0.193 \\
Duration of pain (mo) & $6.4 \pm 14.8$ & $5.0 \pm 19.6$ & 0.362 \\
Initial NRS & $6.5 \pm 1.6$ & $6.9 \pm 1.5$ & 0.471 \\
\hline
\end{tabular}

Values are presented as mean \pm standard deviation or number (\%). NRS: numerical rating scale.

\section{RESULTS}

Of the 201 enrolled patients, $100(49.8 \%)$ were assigned to the control group and 101 (50.2\%) were assigned to the hydromorphone group. Three in the control group and 5 in the hydromorphone group failed to complete follow-up. Demographics and baseline characteristics are shown in Table 1. The average age of patients in the control group was $66.6 \pm 14.9$, including 51 males and 46 females. There were 11 cases with PHN pain in the head and face, 15 cases in the neck and shoulders, 45 cases in the chest and back, and 26 cases in the waist and lower limbs. The mean age of patients in the hydromorphone group was $67.2 \pm 9.6$, including 46 males and 50 females. There were 13 cases with PHN pain in the head and face, 17 cases in the neck and shoulders, 39 cases in the chest and back, and 27 cases in the waist and lower limbs.

The baseline NRS score of the control group was $6.5 \pm$ 1.6, while the baseline NRS score of the hydromorphone group was $6.9 \pm 1.5$, and there was no statistical difference between the two groups $(P=0.114)$. The average duration of PHN pain in the control group was $6.4 \pm 14.8$ months, while that of hydromorphone group was $5.0 \pm 19.6$ months. There was no statistical difference between the two groups $(P=0.093)$. Two weeks after treatment, the mean daily dosage of pregabalin in the hydromorphone group was 225.75 $\pm 74.72 \mathrm{mg}$, while that of the control group was $234.84 \pm$ $75.34 \mathrm{mg}$. There was no statistical difference between the two groups $(P=0.054)$. Two weeks after treatment, the mean daily dosage of hydromorphone in the hydromorphone group was $6.25 \pm 1.98 \mathrm{mg}$.

After treatment, the pain score in both groups was alleviated to varying degrees (Fig. 2). One week, 4 weeks, and 12 weeks after treatment in the control group, the NRS scores were $4.5 \pm 1.4,3.5 \pm 1.3$, and $3.0 \pm 1.0$, while the NRS 


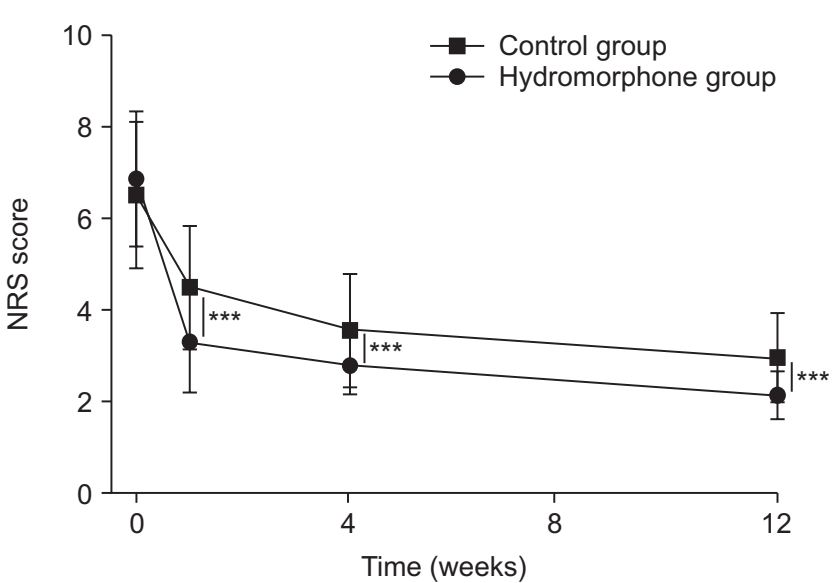

Fig. 2. Effect of treatment on pain scores over time. The decline in the numerical rating scale (NRS) score over time in the hydromorphone group is significantly steeper than that in the control group after treatment $(P<0.001)$. Error bars indicate standard deviation. The asterisks indicate significant differences $(* * * P<0.001)$.

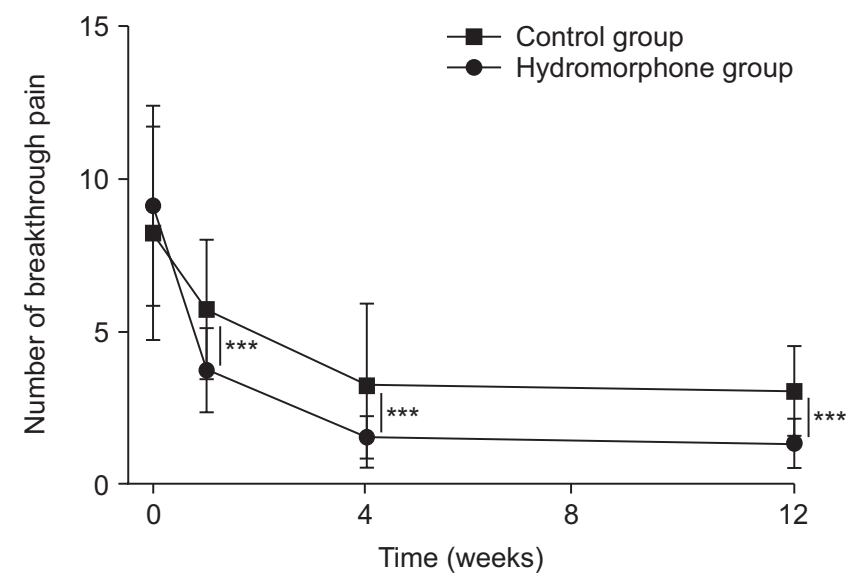

Fig. 3. Effect of treatment on the frequency of breakthrough pain over time. The frequency of breakthrough pain in the hydromorphone group is significantly lower than that in the control group one week and four weeks after treatment $(P<0.001)$. Error bars indicate standard deviation. The asterisks indicate significant differences $(* * * P<0.001)$.

scores for the hydromorphone group were $3.3 \pm 1.1,2.8$ \pm 0.6 , and $2.1 \pm 0.5$, respectively. The difference between the two groups was statistically significant $(P<0.001, P<$ 0.001 , and $P<0.001$, respectively). Moreover, the frequency of breakthrough pain in the hydromorphone group was significantly lower than that in the control group 1 week and 4 weeks after treatment, and the difference was statistically significant $(P<0.001$, Fig. 3$)$. Patients in the hydromorphone group had better pain control.

Pain quality was measured using the total score derived from the validated SFMPQ. After treatment, the SFMPQ score in both groups was alleviated to varying degrees (Fig. 4). Before treatment, 1 week, 4 weeks, and 12 weeks after treatment in the control group, the SFMPQ scores

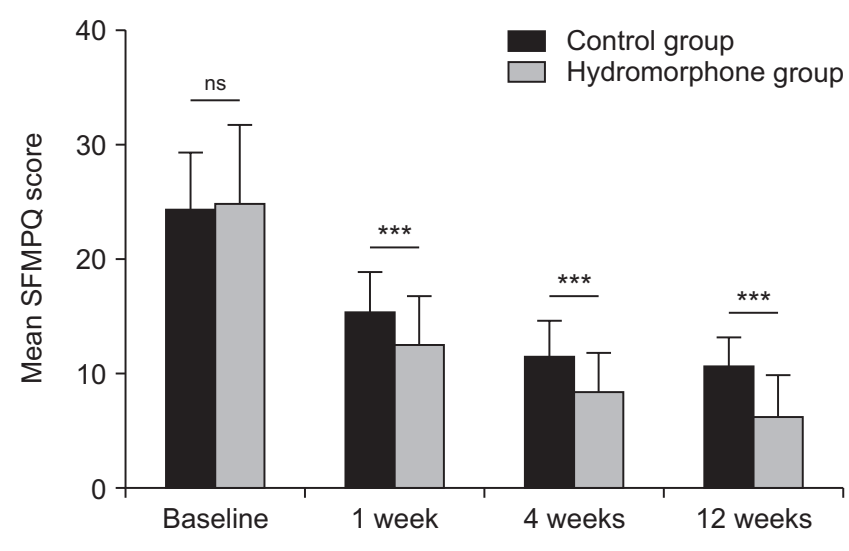

Fig. 4. Mean pain quality scores (Short-Form McGill Pain Questionnaire, SFMPQ) over time. The mean SFMPQ score in the hydromorphone group is significantly decreased compared with the control group after treatment $(P<0.001)$. Error bars indicate standard deviation. The asterisks indicate significant differences $(* * * P<0.001)$. ns: no statistical difference.

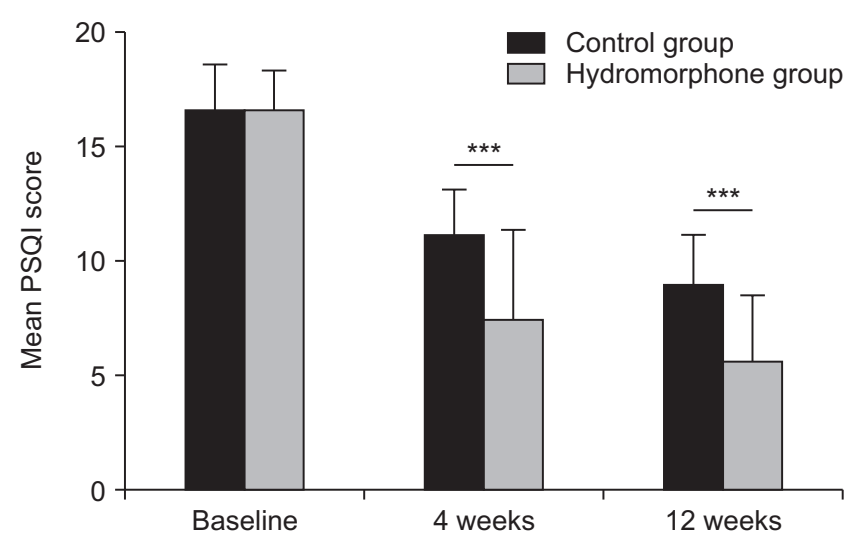

Fig. 5. Pittsburgh sleep quality index (PSQI) in postherpetic neuralgia patients. The mean PSQI score in the hydromorphone group is significantly decreased compared with the control group after treatment $(P<0.001)$. Error bars indicate standard deviation. The asterisks indicate significant differences $(* * * P<0.001)$.

were $24.45 \pm 4.83,15.41 \pm 3.43,11.69 \pm 2.95$, and $10.70 \pm 2.46$, respectively, while the SFMPQ scores for the hydromorphone group were $25.01 \pm 6.72,12.73 \pm 3.97,8.66 \pm 3.11$, and $6.56 \pm 3.34$, respectively. The baseline SFMPQ scores were no statistical difference between the two groups $(P=0.105)$, while the difference between the two groups after treatment was statistically significant $(P<0.001, P<0.001$, and $P<0.001$, respectively). Patients in the hydromorphone group had better pain control.

Sleep quality was measured using the total score derived from the PSQI. After treatment, the PSQI score in both groups was decreased to varying degrees (Fig. 5). Before treatment, 4 weeks, and 12 weeks after treatment in the control group, the PSQI scores were $16.66 \pm 1.88,11.18 \pm$ 1.93 , and $9.02 \pm 2.10$, respectively, while the PSQI scores for 
Table 2. Adverse effects during treatment

\begin{tabular}{|c|c|c|c|c|c|c|}
\hline \multirow{2}{*}{ Symptom } & \multicolumn{3}{|c|}{ Control group $(n=97)$} & \multicolumn{3}{|c|}{ Hydromorphone group $(n=96)$} \\
\hline & 1 week & 4 weeks & 12 weeks & 1 week & 4 weeks & 12 weeks \\
\hline Nausea & $0(0)$ & $0(0)$ & $0(0)$ & $5(5.2)$ & $0(0)$ & $0(0)$ \\
\hline Vomiting & $0(0)$ & $0(0)$ & $0(0)$ & $1(1.0)$ & $0(0)$ & $0(0)$ \\
\hline Pruritus & $0(0)$ & $0(0)$ & $0(0)$ & $0(0)$ & $0(0)$ & $0(0)$ \\
\hline Constipation & $0(0)$ & $0(0)$ & $0(0)$ & $2(2.1)$ & $0(0)$ & $0(0)$ \\
\hline Difficulty passing urine & $0(0)$ & $0(0)$ & $0(0)$ & $2(2.1)$ & $0(0)$ & $0(0)$ \\
\hline Drowsiness & $3(3.1)$ & $2(2.1)$ & $3(3.1)$ & $2(2.1)$ & $1(1.0)$ & $1(1.0)$ \\
\hline Dizziness & $5(5.2)$ & $4(4.1)$ & $3(3.1)$ & $5(5.2)$ & $2(2.1)$ & $0(0)$ \\
\hline Respiratory depression & $0(0)$ & $0(0)$ & $0(0)$ & $0(0)$ & $0(0)$ & $0(0)$ \\
\hline Sweating & $0(0)$ & $0(0)$ & $0(0)$ & 3 (3.1) & $0(0)$ & $0(0)$ \\
\hline Total & $8(8.2)$ & $6(6.2)$ & $6(6.2)$ & $20(20.8)$ & $3(3.1)$ & $1(1.0)$ \\
\hline
\end{tabular}

Values are presented as number (\%).

the hydromorphone group were $16.68 \pm 1.63,7.55 \pm 3.80$, and $5.77 \pm 2.74$, respectively. The baseline PSQI scores showed no statistical difference between the two groups ( $P$ $=0.087$ ), while the difference between the two groups after treatment was statistically significant $(P<0.001$ and $P<$ 0.001 , respectively). Patients in the hydromorphone group had greater sleep improvement.

We evaluated all side effects related to hydromorphone (Table 2). The control group had 3 cases of drowsiness and 5 cases of dizziness one week after treatment, while the hydromorphone group had 5 cases of nausea, 1 case of vomiting, 2 cases of constipation, 2 cases of urinary retention, 2 cases of drowsiness, 5 cases of dizziness, and 3 cases sweating. The control group had 2 cases of drowsiness and 4 cases of dizziness four weeks after treatment, while the hydromorphone group had 1 case of drowsiness and 2 cases of dizziness four weeks after treatment. The control group had 3 cases of drowsiness and 3 cases of dizziness 12 weeks after treatment, while the hydromorphone group had 1 case of drowsiness 12 weeks after treatment. No patient experienced respiratory depression in either group. Adverse reactions were transient and improved after symptomatic treatment. There were no significant differences in side effects after treatment between the two groups $(P=0.144)$.

\section{DISCUSSION}

This was a multicenter, randomized controlled study. The results showed that IV PCA hydromorphone combined with oral pregabalin is more effective in controlling pain in patients with PHN than oral pregabalin alone. The difference between treatments was observed at 1 week after treatment and lasted for 12 weeks.

Hydromorphone, a semisynthetic derivative of mor- phine, is five to seven times more effective in relieving pain than morphine. Although hydromorphone has been used to treat severe pain since 1975, its use in PHN has not been reported. Opioids are often administered according to a schedule rather than on demand, which can lead to poor pain relief in patients with PHN.

In this study, we treated PHN with IV PCA in order to better control breakthrough pain. Breakthrough pain has a significant negative impact on the overall pain degree of PHN patients, and effective management of breakthrough pain is essential. In this study, patients in the hydromorphone group had better pain control after IV PCA hydromorphone, which may be related to timely and self-administration when the patient was in pain. Hydromorphone usually works 15 minutes after intravenous administration [16], and our research shows that patients use a daily hydromorphone dosage of 2-8 $\mathrm{mg}$. After one week of IV PCA hydromorphone combined with oral pregabalin (150-300 mg/d), the pain was significantly reduced. Four weeks and 12 weeks after treatment, the pain in the hydromorphone group was also significantly improved compared with the control group. Although hydromorphone was only used for two weeks, it rapidly reduced the degree of pain, and the pain did not rebound significantly after withdrawal.

In addition to assessing the pain of patients with PHN through NRS and SFMPQ scores, we also evaluated the quality of sleep through the PSQI. Our study showed that the sleep quality of the patients in the hydromorphone group had a significant improvement at one week after treatment, and the sleep quality in the 4 weeks and 12 weeks after treatment was better than that in the control group. Our study showed that IV PCA hydromorphone can not only improve the pain of patients with PHN, but also improve the daily life of patients, especially the quality of sleep. 
Our study found that the control group had adverse reactions such as drowsiness and dizziness, while the hydromorphone group had nausea, vomiting, constipation, urinary retention, drowsiness, dizziness, and sweating. No respiratory depression occurred in either group of patients. Adverse reactions may be related to the use of opioids, but the adverse reactions are transient and improved after symptomatic treatment. The difference in adverse reactions between the two groups after treatment was not statistically significant, indicating that the use of hydromorphone did not significantly increase the probability of adverse reactions.

The strength of this study is the design of a multicenter, randomized control to reduce possible bias, while using multiple methods to assess pain and quality of life in patients with PHN. However, the limitation of the analysis is that it was only followed up for 12 weeks, and no longer follow-up was performed to evaluate hydromorphone's longer-term safety and effectiveness.

In summary, this study shows that IV PCA hydromorphone combined with oral pregabalin is more effective for patients with PHN than oral pregabalin alone. IV PCA hydromorphone provides rapid onset of pain relief and is worthy of clinical promotion and application.

\section{CONFLICT OF INTEREST}

The authors confirm that there are no known conflicts of interest associated with this publication and there has been no significant financial support for this work that could have influenced its outcome.

\section{FUNDING}

This work was supported by grants from Yichang Humanwell Pharmaceutical Project (No. 20180928007), the National Natural Science Foundation of China (No. 81801100), the Natural Science Foundation of Jiangsu Province (No. BK20180130), Medical Science and Technology Development Project of Nanjing (No.YKK17072).

\section{ORCID}

Ying Huang, https://orcid.org/0000-0003-3501-4739 Chenjie Xu, https://orcid.org/0000-0001-5942-7015 Tao Zeng, https://orcid.org/0000-0003-1497-3279 Zhongming Li, https://orcid.org/0000-0002-6900-3037 Yanzhi Xia, https://orcid.org/0000-0003-0315-7698 Gaojian Tao, https://orcid.org/0000-0002-3556-096X
Tong Zhu, https://orcid.org/0000-0001-5901-4712

Lijuan Lu, https://orcid.org/0000-0002-5655-0971

Jing Li, https://orcid.org/0000-0003-4687-825X

Taiyuan Huang, https://orcid.org/0000-0002-5932-1437

Hongbo Huai, https://orcid.org/0000-0001-6851-7266

Benxiang Ning, https://orcid.org/0000-0002-4439-3307

Chao Ma, https://orcid.org/0000-0002-7911-9084

Xinxing Wang, https://orcid.org/0000-0003-4057-3105

Yuhua Chang, https://orcid.org/0000-0001-8948-3595

Peng Mao, https://orcid.org/0000-0002-7861-653X

Jian Lin, https://orcid.org/0000-0001-7692-3774

\section{REFERENCES}

1. Tsai CC, Chou YY, Chen YM, Tang YJ, Ho HC, Chen DY. Effect of the herbal drug guilu erxian jiao on muscle strength, articular pain, and disability in elderly men with knee osteoarthritis. Evid Based Complement Alternat Med 2014; 2014: 297458.

2. Watson CP. A new treatment for postherpetic neuralgia. $\mathrm{N}$ Engl J Med 2000; 343: 1563-5.

3. Schmader KE. Epidemiology and impact on quality of life of postherpetic neuralgia and painful diabetic neuropathy. Clin J Pain 2002; 18: 350-4.

4. Johnson RW, Dworkin RH. Treatment of herpes zoster and postherpetic neuralgia. BMJ 2003; 326: 748-50.

5. Johnson RW, Bouhassira D, Kassianos G, Leplège A, Schmader KE, Weinke T. The impact of herpes zoster and postherpetic neuralgia on quality-of-life. BMC Med 2010; 8: 37.

6. Gnann JW Jr, Whitley RJ. Clinical practice. Herpes zoster. N Engl J Med 2002; 347: 340-6.

7. Hansson PT, Dickenson AH. Pharmacological treatment of peripheral neuropathic pain conditions based on shared commonalities despite multiple etiologies. Pain 2005; 113: 251-4.

8. Baron R, Wasner G. Prevention and treatment of postherpetic neuralgia. Lancet 2006; 367: 186-8.

9. Quigley C. Hydromorphone for acute and chronic pain. Cochrane Database Syst Rev 2002; 1: CD003447.

10. Sarhill N, Walsh D, Nelson KA. Hydromorphone: pharmacology and clinical applications in cancer patients. Support Care Cancer 2001; 9: 84-96.

11. Mahler DL, Forrest WH Jr. Relative analgesic potencies of morphine and hydromorphone in postoperative pain. Anesthesiology 1975; 42: 602-7.

12. Dworkin RH, O'Connor AB, Backonja M, Farrar JT, Finnerup NB, Jensen TS, et al. Pharmacologic management of neuropathic pain: evidence-based recommendations. Pain 2007; 132: 237-51.

13. Rauck RL, Irving GA, Wallace MS, Vanhove GF, Sweeney M. Once-daily gastroretentive gabapentin for postherpetic 
neuralgia: integrated efficacy, time to onset of pain relief and safety analyses of data from two phase 3, multicenter, randomized, double-blind, placebo-controlled studies. J Pain Symptom Manage 2013; 46: 219-28.

14. Melzack R. The short-form McGill Pain Questionnaire. Pain 1987; 30: 191-7.

15. Raskind MA, Peskind ER, Chow B, Harris C, Davis-Karim A,
Holmes HA, et al. Trial of prazosin for post-traumatic stress disorder in military veterans. N Engl J Med 2018; 378: 507-17.

16. Chang AK, Bijur PE, Lupow JB, Gallagher EJ. Randomized clinical trial of the $2 \mathrm{mg}$ hydromorphone bolus protocol versus the " $1+1$ " hydromorphone titration protocol in treatment of acute, severe pain in the first hour of emergency department presentation. Ann Emerg Med 2013; 62: 304-10. 